Article

\title{
Correlative Light and Transmission Electron Microscopy Showed Details of Mitophagy by Mitochondria Quality Control in Propionic Acid Treated SH-SY5Y Cell
}

\author{
Minkyo Jung ${ }^{1,+}$, Hyosun Choi ${ }^{1,2,+}$, Jaekwang Kim $^{3}$ and Ji Young Mun ${ }^{1, *}$ \\ 1 Neural Circuit Research Group, Korea Brain Research Institute, Daegu 41062, Korea; j0312@kbri.re.kr (M.J.); \\ hyokchoi0123@gmail.com (H.C.) \\ 2 BK21 Plus Program, Department of Senior Healthcare, Graduate School, Eulji University, \\ Daejeon 34824, Korea \\ 3 Dementia Research Group, Korea Brain Research Institute, Daegu 41062, Korea; kim_jaekwang@kbri.re.kr \\ * Correspondence: jymun@kbri.re.kr \\ + These authors contributed equally to this work.
}

Received: 25 August 2020; Accepted: 27 September 2020; Published: 29 September 2020

check for updates

\begin{abstract}
Propionic acid is a metabolite of the microbiome and can be transported to the brain. Previous data show that propionic acid changes mitochondrial biogenesis in SH-SY5Y cells and induces abnormal autophagy in primary hippocampal neurons. Maintaining mitochondrial function is key to homeostasis in neuronal cells, and mitophagy is the selective autophagy involved in regulating mitochondrial quality. Monitoring mitophagy though light microscopy or conventional transmission electron microscopy separately is insufficient because phases of mitophagy, including autophagosome and autolysosome in nano-resolution, are critical for studies of function. Therefore, we used correlative light and electron microscopy to investigate mitochondrial quality in SH-SY5Y cells after propionic acid treatment to use the advantages of both techniques. We showed, with this approach, that propionic acid induces mitophagy associated with mitochondrial quality.
\end{abstract}

Keywords: propionic acid; autophagy; mitophagy; correlative light and electron microscopy (CLEM)

\section{Introduction}

Short-chain fatty acids (SCFAs) such as acetic, propionic, and butyric acid are by-products of fermentation of dietary fiber by the gut microbiome [1]. Microbe-derived metabolites can cross the blood-brain barrier and affect the neurons. As the relationship between gut microbiome and the brain, gut-brain axis, has become interesting, SCFAs have attracted increasing attention. Propionic acid (PPA) is increased in stools from patients with autistic spectrum disorder, and prenatal exposure to PPA causes significant impairment of the social behavior of neonatal rat offspring [2]. Further, PPA administration to rodents alters expression of genes associated with neurotransmitters, neuronal cell adhesion molecules, inflammation, oxidative stress, lipid metabolism, and mitochondrial function [3-5]. Conversely, decreases in PPA are reported in patients with multiple sclerosis, an autoimmune and neurodegenerative disease [6]. One important cellular process negatively affected by PPA is mitochondrial function [7]. Rats exposed to PPA show mitochondrial dysfunction and an increase in free acyl-carnitine, a factor for the transport of long-chain fatty acids into mitochondria [8]. PPA and butyric acid also induce autophagy in human colon cancer cells that limits apoptosis, and inhibition of autophagy potentiates SCFA-induced apoptosis [9]. As our previous data indicated that PPA induces abnormal autophagy in PPA-treated hippocampal neuron [10], we 
investigated the relationship between mitochondrial defects and the regulation of mitochondrial quality though mitophagy.

Mitophagy is a selective degradative process responsible for removing damaged mitochondria to maintain cytoplasmic homeostasis [11]. Mitochondrial dysfunction is involved in various neurodegenerative or neurodevelopmental diseases [12]. Once mitophagy is initiated, a balance between autophagosome formation and autophagic degradation is necessary. Thus, accumulation of autophagosomes and disruption of the autophagic process in neurons is associated with disease [13]. Until now, conventional techniques for analysis of mitophagy have been based on immunofluorescence staining and immunoblotting of several specific mitochondrial proteins, qPCR for mitochondrial DNA copy number, and nano-resolution imaging using transmission electron microscopy (TEM). TEM is a direct imaging method for the early stage of mitophagy, which is the starting point of engulfing mitochondria and early autophagosome showing specific mitochondrial structures such as cristae [14,15]. However, the assessment of the late phases of mitophagy requires specific imaging techniques.

Recently, the engineering of two fluorescent proteins (mCherry-GFP-mito, and mt-Keima) has permitted monitoring of the status of mitophagy in live cells. These reporters change the fluorescence profile in response to $\mathrm{pH}$ changes. For example, the excitation wavelength for $\mathrm{mt}-\mathrm{Keima}$ is 488 at neutral $\mathrm{pH}$ and 561 at acidic $\mathrm{pH}$ for late mitophagy observed in the lysosome [16,17]. mCherry-GFP-mito protein, fused to a mitochondrial targeting sequence of a mitochondrial protein, such as the outer mitochondrial membrane (OMM) protein FIS1 (comprising amino acids 101-152) [18] can be used to detect mitophagy. The mitochondrial network can be seen as a green fluorescence, and mitochondria delivered to lysosomes show as a red color after mitophagy. This is because mCherry fluorescence is stable, but GFP fluorescence is quenched in the acidic condition. However, these tools do not allow the monitoring of all phases of mitophagy. To analyze the entire dynamic phase of autophagy regarding mitophagy, TEM is employed to classify the specific type of autophagy including phagophore, autophagosome, and autolysosome in high resolution $[19,20]$. Therefore, we analyzed structural changes by TEM to study specific stages of autophagy that are more tightly linked with the mechanisms of mitophagy dysfunction. Thus, correlative light and electron microscopy (CLEM) is an effective method to analyze mitophagy or autophagic pathways [21]. Because the Keima protein is incompatible with fixation [22], we used GFP and mCherry conversion depending on the $\mathrm{pH}$ level of lysosomes to investigate details of various steps of mitophagy in nano-resolution though electron microscopy (EM). The CLEM technique of overlaying two images from fluorescence and EM makes the investigation of all phases of mitophagy possible. Evans et al. suggested that CLEM can open new avenues using light-up through (fluorescent) dyes in the dark by EM observation [23]. Thus, we applied CLEM to study mitophagy after PPA treatment.

\section{Materials and Methods}

\subsection{Cell Culture}

SH-SY5Y control cells, obtained from Dr. Kim H.J (KBRI), and the tandem mCherry-GFP tag fused to FIS1 stable SH-SY5Y cells, a kind gift from Dr. Ian G. Ganley (University of Dundee, Dundee, UK) [18], were grown in normal culture conditions with DMEM/F12 (ThermoFisher, Waltham, MA, USA) supplemented with 15\% fetal bovine serum (ThermoFisher, USA), 100 units $/ \mathrm{mL}$ of penicillin, and $100 \mathrm{ug} / \mathrm{mL}$ of streptomycin (ThermoFisher, USA) at $37^{\circ} \mathrm{C}$ in a humidified $5 \% \mathrm{CO}_{2}$ atmosphere. The tandem mCherry-GFP tag fused to FIS1 stable SH-SY5Y cells were selected with $500 \mu \mathrm{g} / \mathrm{mL}$ of hygromycin (Sigma, St. Louis, MO, USA) and a stable pool was used for experiments. 


\subsection{Viability Assay}

SH-SY5Y cells were plated and treated with PPA $(0.1,1,2,6$, and $12 \mathrm{mM}$, Sigma, St. Louis, MO, USA) in 96-well plates for 48 and $72 \mathrm{~h}$ Approximately $10 \mu \mathrm{L}$ of CCK-8 (Dojindo, Kumamoto, Japan) was added to cells, and the optical density (OD) value was measured at $450 \mathrm{~nm}$.

\subsection{Immunocytochemistry}

SH-SY5Y cells were grown on coverslips and treated with $1 \mathrm{mM}$ PPA (Sigma, USA) for $72 \mathrm{~h}$ Cells were fixed with $1 \%$ paraformaldehyde (EMS, Hatfield, PA, USA) in phosphate-buffered saline (PBS, Welgene, Gyeongsangbuk-do, Gyeongsan-si, Korea) containing 4\% sucrose for $5 \mathrm{~min}$ at room temperature. Primary antibodies against LC3A/B (\#12741, Cell Signaling, Danvers, MA, USA) were added with blocking solution containing $0.1 \%$ gelatin, $0.3 \%$ Triton $\mathrm{X}-100,16 \mathrm{mM}$ sodium phosphate, and $450 \mathrm{mM} \mathrm{NaCl}$, and cells were incubated overnight at $4{ }^{\circ} \mathrm{C}$. After being washed with PBS, coverslips were incubated with Alexa Fluor488 (\#4412, Cell Signaling, USA)-conjugated secondary antibodies for $1 \mathrm{~h}$ at room temperature and then again washed with PBS. Next, coverslips were mounted with a mounting medium (H-1000, Vector Laboratories, Burlingame, CA, USA) and were imaged with fluorescence microscopy (Nikon, Tokyo, Japan) using a $488 \mathrm{~nm}$ fluorescence filter.

\subsection{Transmission Electron Microscopy for Quantifying Autophagic Elements}

SH-SY5Y cells were treated with $1 \mathrm{mM}$ of PPA for $72 \mathrm{~h}$ and then fixed with $2.5 \%$ glutaraldehyde $/ 2 \%$ paraformaldehyde solution for $2 \mathrm{~h}$ Fixed cells were then post-fixed with $2 \%$ osmium tetroxide (EMS, USA) for $2 \mathrm{~h}$ at $4{ }^{\circ} \mathrm{C}$, and the block was stained in $0.1 \mathrm{mg}$ thiocarbohydrazide (TCH, TCI, Tokyo, Japan) in $10 \mathrm{~mL}$ distilled water and 1\% uranyl acetate (EMS, USA) and dehydrated with a graded ethanol series. The samples were then embedded with an EMBed-812 embedding kit (EMS, USA). The embedded samples were sectioned $(60 \mathrm{~nm})$ with an ultramicrotome (Leica, Wetzlar, Germany), and the sections were then viewed on a Tecnai G2 transmission electron microscope (Thermofisher) at $120 \mathrm{kV}$. The numbers of autophagosomes and autolysosomes per cell were assessed.

\subsection{Correlative Light and Electron Microscopy}

CLEM was performed as previously described [23]. The mCherry-GFP tag fused to FIS1 stable SH-SY5Y cells were grown in $35 \mathrm{~mm}$ glass grid-bottomed culture dishes to $50-60 \%$ confluency. Cells with or without $1 \mathrm{mM}$ or $2 \mathrm{mM}$ PPA treatment were stained with $100 \mathrm{nM}$ LysoTracker (LysoTracker Blue DND-22, Thermofisher, USA) for $15 \mathrm{~min}$ and then imaged under a confocal light microscope (Ti-RCP, Nikon, Japan), and after $24 \mathrm{~h}$ treatment of PPA, cells were fixed with $1 \%$ glutaraldehyde and $1 \%$ paraformaldehyde in $0.1 \mathrm{M}$ cacodylate solution ( $\mathrm{pH}$ 7.0). After being washed, cells were dehydrated with a graded ethanol series and infiltrated with an embedding medium. After embedment, $60 \mathrm{~nm}$ sections were cut horizontally to the plane of the block (UC7; Leica Microsystems, Germany) and were mounted on copper slot grids with a specimen support film. Sections were stained with uranyl acetate and lead citrate. The cells were observed at $120 \mathrm{kV}$ in a Tecnai G2 microscope (ThermoFisher, USA). Confocal micrographs were produced as high-quality large images using PhotoZoom Pro 8 software (Benvista Ltd., Houston, TX, USA). Enlarged fluorescence images were fitted to the electron micrographs using the Image J BigWarp program.

\subsection{Measurement of Mitophagy}

The mCherry-GFP tag fused to FIS1 stable SH-SY5Y cells $\left(5 \times 10^{4}\right.$ cells/well $)$ was grown in $35 \mathrm{~mm}$ glass-bottomed culture dishes (MatTEK, Ashland, MA, USA) and treated with 1 and $2 \mathrm{mM}$ PPA. Parallel incubation of cells without PPA was used for control. Measurement of mitophagy has been described previously [16]. Briefly, quantitation was performed for five fields of view for each group. Red-alone puncta were defined as round structures found only in the red channel with no 
corresponding structure in the green channel. Quantitative data were collected by manually counting all red-only puncta within each cell for each field of view.

\section{Results}

The viability of SH-SY5Y cells after treatment with PPA showed that optimal concentrations of PPA were less than $2 \mathrm{mM}$. Viability was assessed with CCK-8 assays after treatment with concentrations of $0,0.1,1,2,6$, and $12 \mathrm{mM}$. Viability was significantly decreased in response to $2 \mathrm{mM}$ after $48 \mathrm{~h}$ incubation with PPA (Figure 1A). After $72 \mathrm{~h}$, treatment with $1 \mathrm{mM}$ PPA a significant change was also shown (Figure 1B). Therefore, we used $1 \mathrm{mM}$ PPA for $72 \mathrm{~h}$ to assess autophagy in SH-SY5Y cells. The number of LC3 puncta in PPA-treated cells was increased, as shown by immunofluorescence (Figure 1C). The number of LC3 puncta was $2.9 \pm 0.28$, compared with $1.8 \pm 0.3293$ for untreated cells (Figure 1D). No difference in the intensity of LC3 puncta was observed.

A

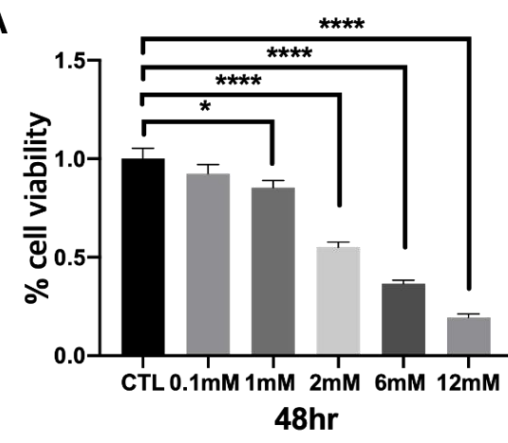

B

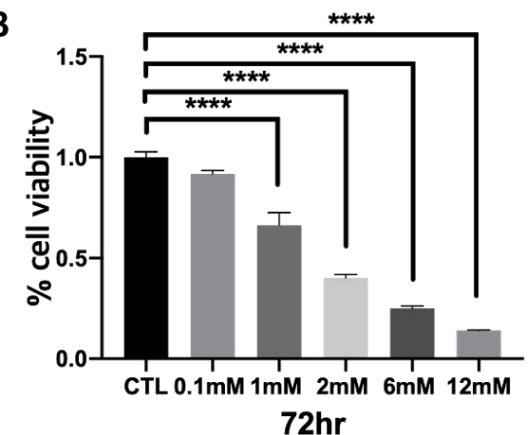

C

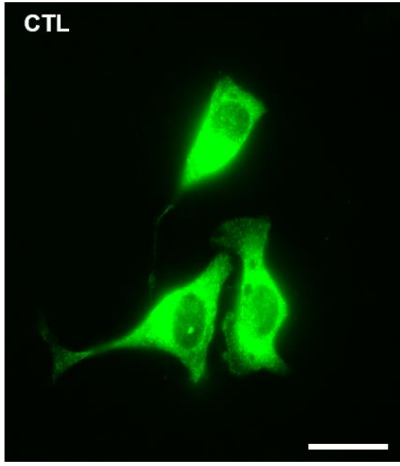

D

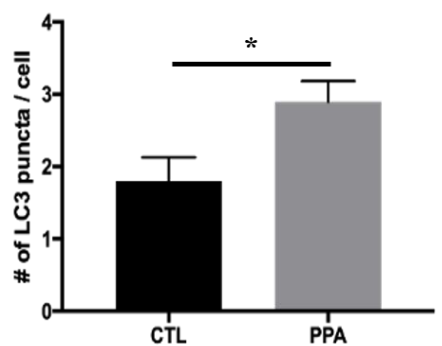

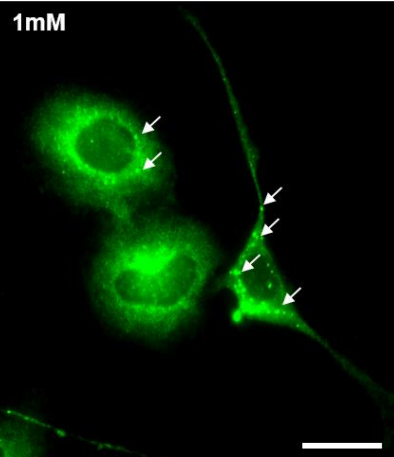

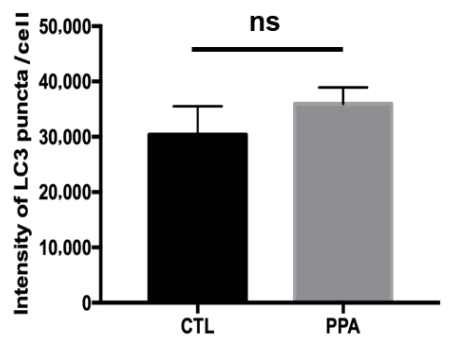

Figure 1. Viability assay and increase of of LC3 level in SH-SY5Y cells after propionic acid (PPA) treatment. The cells were treated with PPA for (A) $48 \mathrm{~h}$ and (B) $72 \mathrm{~h}$ Statistical analysis used a two- way ANOVA. Results are presented as mean \pm SEM. When the concentration reached 2-12 mM, cell viability was significantly reduced. (C) Representative immunofluorescence images showing LC3 puncta in SH-SY5Y control cells and PPA-treated cells. The white scale bar is $50 \mu \mathrm{m}$. (D) Numbers of LC3 puncta and LC3 puncta intensity from images in $(\mathbf{A})(n=5)$, illustrating a significant increase of number of LC3 puncta following treatment with $1 \mathrm{mM}$ PPA. Statistical analysis used a one-way ANOVA. Results are presented as mean \pm SEM. ${ }^{*} p<0.05,{ }^{* * * *} p<0.0001$.

We further analyzed the number of autophagosomes and autolysosomes in control and PPA-treated cells using TEM images. The numbers of both organelles were increased in PPA-treated cells. The numbers of autophagosomes per cell were $6.1 \pm 0.7371$ and $1.9 \pm 0.4333$ for treated and control cells, respectively (Figure 2A). Similar results for autolysosomes were found: $6.6 \pm 1.067$ for PPA-treated cells and $1.9 \pm 0.5667$ for untreated cells (Figure 2B). 
(A)

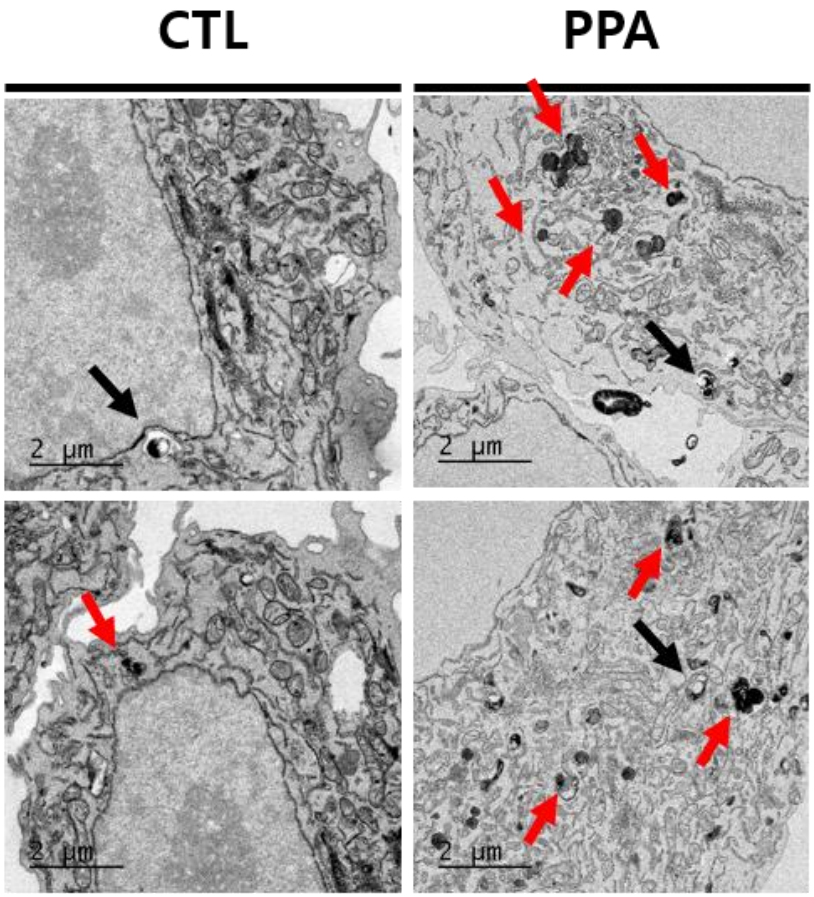

(B)

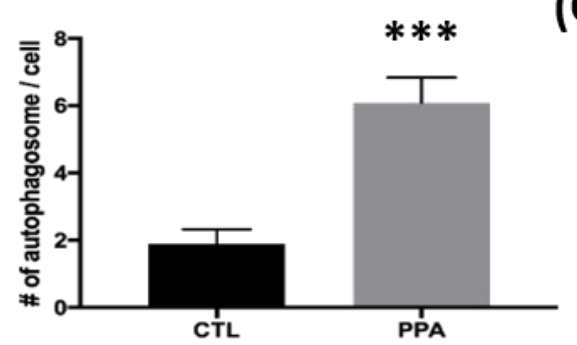

(C)

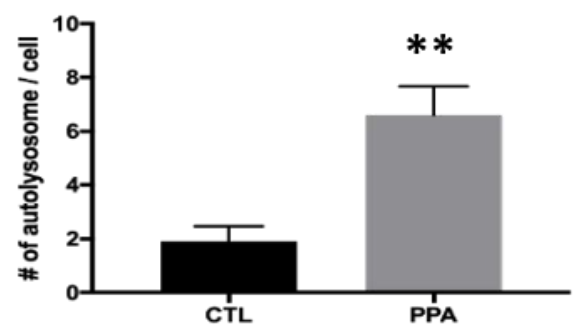

Figure 2. The increase in autophagy following PPA treatment in SH-SY5Y cells using TEM. (A) Representative TEM images showing autophagy in control SH-SY5Y cells and PPA-treated cells. The black arrow indicates autophagosomes, and red arrows indicate autolysosomes. (B and C) Numbers of autophagosomes and autolysosomes from images in $(\mathbf{A})(\mathrm{n}=10)$. TEM analysis shows that numbers of both autophagosomes and autolysosomes increase in cells treated with $1 \mathrm{mM}$ PPA. Statistical analysis used a one-way ANOVA. Results are presented as mean \pm SEM. ${ }^{* *} p<0.01,{ }^{* * *} p<0.0005$.

PPA was reported as a small molecule leading to mitochondrial dysfunction [7]. Therefore, we used the tandem mCherry-GFP tag fused to FIS1-stable SH-SY5Y cells to confirm the induction of mitophagy by PPA treatment. GFP and mCherry show green and red fluorescence, respectively, with the former specific for mitochondria and the latter for mitophagy (Figure 3A). After treatment with 1 or $2 \mathrm{mM}$ PPA, the number of mCherry red puncta increased 4.6 times, indicating the induction of mitophagy in treated cells (Figure 3B). 

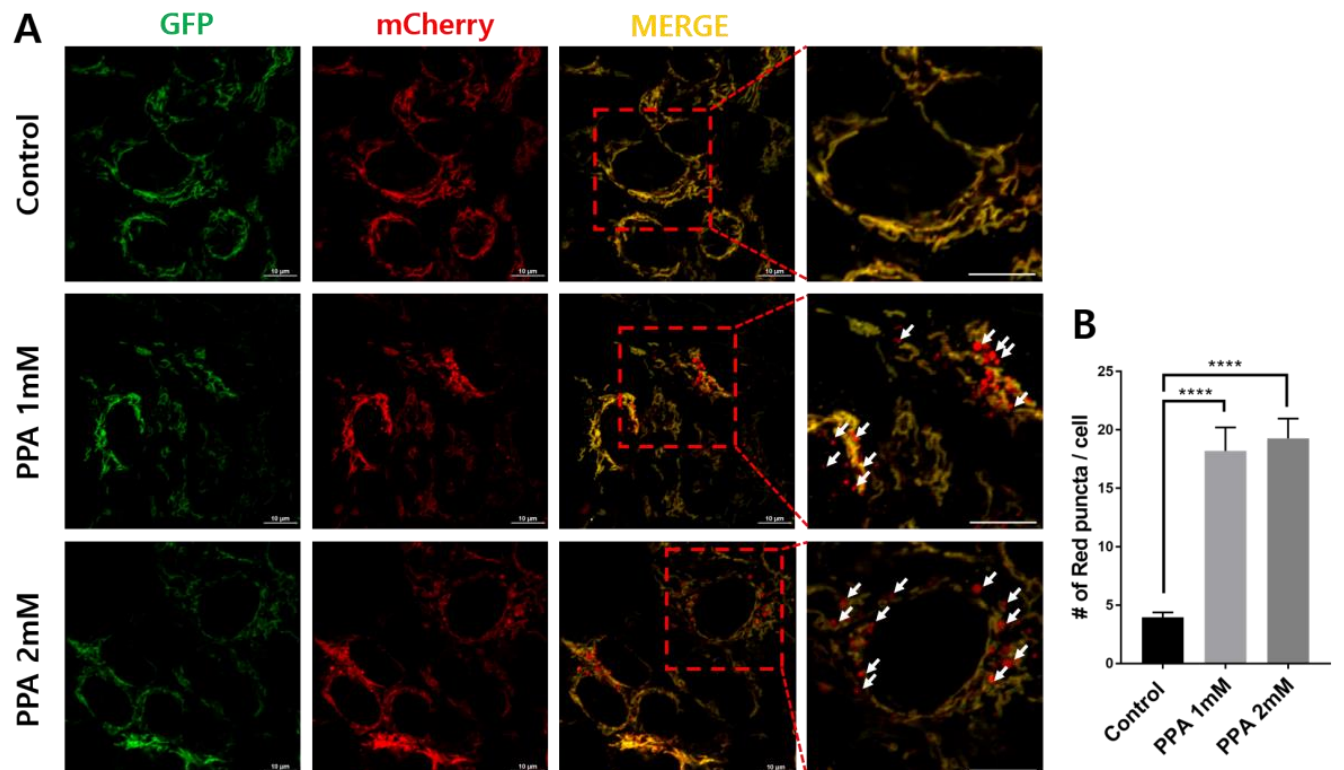

Figure 3. Mitophagy assays in SH-SY5Y cells following treatment with 1 and $2 \mathrm{mM}$ PPA for $24 \mathrm{~h}$. (A) mCherry-GFP-FIS1101-152 stably expressing cells were subjected to (1) control, (2) $1 \mathrm{mM}$ PPA, and (3) $2 \mathrm{mM}$ PPA for $24 \mathrm{~h}$. (B) Numbers of red puncta (mitophagy) per cell ( $\mathrm{n}>40)$. Statistical analysis used a one-way ANOVA. Results are presented as mean \pm SEM. ${ }^{* * * *} p<0.0001$.

We employed CLEM to confirm the ultrastructure of red puncta (Figure 4). Live cells were imaged using confocal microscopy treatment with PPA after $24 \mathrm{~h}$. Images were then aligned with stitched TEM images of the same cells. Almost healthy mitochondria showed the green fluorescence of GFP, and some red puncta were co-localized with LysoTracker (Figure 4A). In control cells, LysoTracker-positive structures (blue) are seen contacting red puncta (Figure 4A, white dot line box, and enlarged images). In the PPA treatment cells, damaged round mitochondria show high electron density in the electron micrographs and are co-localized with LysoTracker, suggesting mitophagy (Figure 4B).

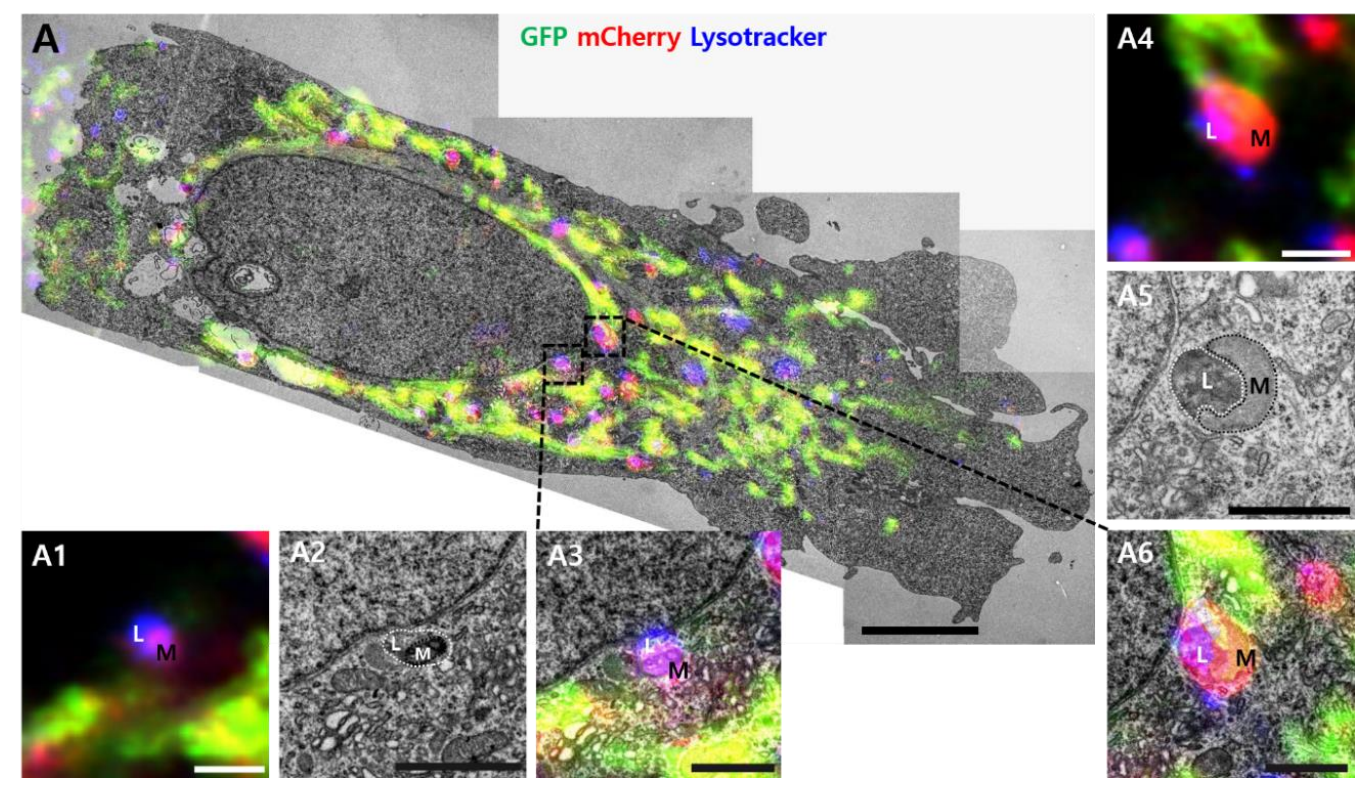




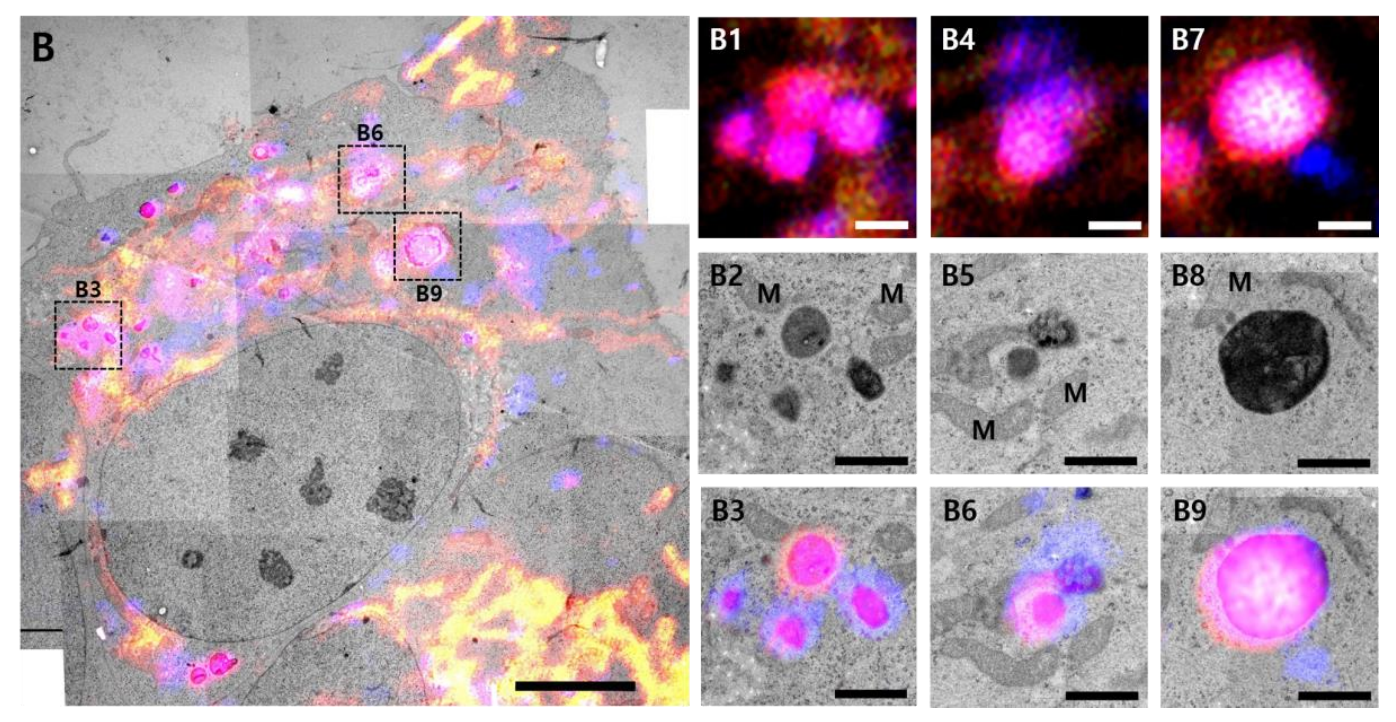

Figure 4. Correlative light and electron microscopy. Correlative confocal and electron microscope images of (A) co-localized mCherry-GFP-FIS1101-152 in SH-SY5Y cells or (B) cells treated with $2 \mathrm{mM}$ PPA. Yellow indicates co-localization of GFP and mCherry signals. Magenta indicates co-localization of mCherry and LysoTracker. Multiple TEM images were taken at 1700× magnification. Images were stitched for a large field of view at higher resolution. The black dot line box indicates structures corresponding to magenta fluorescent puncta on fluorescence images. The structures showed the morphology of mitophagy, as demonstrated by the black dot line box shown at higher magnification in the inserted images (A1-A6 and B1-B9). L, lysosome; M, mitochondria. Size bar in A and $\mathrm{B}=5 \mu \mathrm{m}, \mathrm{A} 1 \sim \mathrm{A} 6=1 \mu \mathrm{m}, \mathrm{B} 1 \sim \mathrm{B} 9=1 \mu \mathrm{m}$.

\section{Discussion}

Mitochondria are continuously replenished. As new mitochondria are produced, dysfunctional organelles are removed by autophagy-mediated degradation though mitophagy [24]. There are several control or repair systems for mitochondrial structure and function maintaining essential energy metabolism. Oxidatively damaged proteins in the mitochondrial outer membrane can be degraded by the ubiquitin-proteasome system. When damage is more extensive, e.g., through exposure to elevated reactive oxygen species (ROS) or aging, mitochondria are sequestered by autophagosome and fused with lysosome for degradation. It is called mitophagy. The extent of mitophagy in neurites is influenced by various factors related to mitochondrial function, and the contribution of mitophagy to mitochondrial function in soma or neurites is critical to understanding the regulation of energy metabolism in these cells [25]. Our previous work shows that PPA induces defects in mitochondria [6] and autophagy [10]. In this study, we investigated the relationship between mitochondrial dysfunction and increased autophagy.

Some mitochondrial toxins, including rotenone, concomitantly activate autophagy, including mitophagy. Like rotenone, PPA-treated cells showed elevated autophagic sequestration of mitochondria. More prominent LC3 signals (Figure 1C) and LC3-II/ $\beta$-actin ratios (data not shown) indicate change of autophagy in PPA-treated cells. Several reports that focus on mitochondrial function in PPA-treated cells are available. Kim et al. showed an increase of mitochondrial copy number and expression of PGC-1a, COX4, SIRT3, and, TFAM (mitochondrial biogenesis-related proteins) after PPA treatment of SH-SY5Y cells [7]. Dysfunction of mitochondria caused by RNA interference-mediated knockdown of peroxisome proliferator-activated receptor- $\gamma$ coactivator $1 \alpha$ (PGC- $1 \alpha)$ in neurons showed abnormal synapse formation in developing neural circuits and failure to maintain synapses in the hippocampus of adults [26]. Induction of mitochondrial biogenesis following expression of PGC- $1 \alpha$ is stimulated by brain-derived neurotrophic factor, which can be modulated by changes of SCFAs in the brain [27]. PPA-induced mitochondrial dysfunction suggests a mechanism for neurotoxicity. El-Ansary et 
al. showed PPA-induced neurotoxicity in rat pups though depletion of gamma-aminobutyric acid and serotonin. [28] Frye et al. showed oxidative stress after PPA exposure, and Alfawaz et al. showed that factors related to mitochondria, such as carnosine, N-acetylcysteine, and vitamin $D$, can rescue neurotoxicity caused by PPA in rat pups [29]. The protective effect of carnosine ( $\beta$-alanyl-L-histidine) is related to autophagy and causes a decrease in Drp-1 expression. Further, treatment with $\mathrm{N}$-acetylcysteine shows inhibition of Atg32-dependent mitophagy [29]. In PPA-treated SH-SY5Y cells in the present study, TEM analysis shows an increase in numbers of both autophagosomes and autolysosomes, which reflects properly functioning autophagy flux (Figure 2).

Several technical challenges using fluorescence and biochemical techniques to analyze autophagic processes, including mitophagy, are recognized $[17,30]$. Such challenges were met in this study using CLEM techniques to monitor autophagy for cellular homeostasis due to mitochondrial dysfunction in PPA-treated SH-SY5Y cells. The tandem mCherry-GFP tag fused to FIS1 was used in our approach. Red fluorescence of mCherry increased in PPA-treated cells, suggesting increased mitophagy, and the green fluorescent of GFP in mitochondria did not change significantly (Figure 3). CLEM confirmed the ultrastructure associated with these colors as mitochondria and mitophagy (Figure 4). There is a mismatch between some healthy mitochondrial fluorescence signal (yellow color) and EM images due to technical limitations of the TEM based CLEM method (Supplementary Figure S1 A1 and B1). It is difficult to accurately match the $\mathrm{Z}$ axis of the optical section (LM) and the physical section (EM), since LM and EM image thicknesses are different. Due to the difference, there is more information in the fluorescence micrograph (LM: $300 \mathrm{~nm}$ optical thickness, EM: $60 \mathrm{~nm}$ physical thickness). Although there is some technical limitation, the damaged mitochondria are well correlated with the lysotracker (Supplementary Figure S1, black arrow). Thus, observations depicted in Figures 3 and 4 indicate that increased autophagy shown in Figures 1 and 2 is mitophagy.

\section{Conclusions}

Changes in mitophagy under stress condition is associated with pathological conditions, including neurodegenerative diseases and myopathies. Therefore, identifying mitophagy modulators and understanding their mechanisms of action will provide critical insight into neurodegenerative diseases. We confirmed that mitophagy was induced by PPA treatment in SH-SY5Y cells. CLEM is a useful technique for monitoring mitophagy in cells under stress. Various stages of mitophagy, including initiation of autophagy, vesicle completion, lysosome fusion, and degradation of mitochondria in lysosomes, can be monitored in CLEM, if the time points in such studies are adequately controlled. CLEM might also be applied to study structural changes of other cellular organelles.

Supplementary Materials: The following are available online at http://www.mdpi.com/1996-1944/13/19/4336/s1, Figure S1: Correlative confocal and electron microscope images of the control (A) and $2 \mathrm{mM}$ PPA treated cell (B).

Author Contributions: Conceptualization, M.J., H.C., and J.Y.M.; methodology, M.J., H.C., and; formal analysis, M.J., H.C.; investigation, M.J., H.C., and J.Y.M.; resources, J.K.; data curation, M.J., H.C.; writing-original draft preparation, J.Y.M.; writing-review and editing, M.J., H.C., J.K., and J.Y.M.; visualization, M.J., H.C.; supervision, J.Y.M.; project administration, J.Y.M.; funding acquisition, J.K. and J.Y.M. All authors have read and agreed to the published version of the manuscript.

Funding: This research was supported by the National Research Foundation of Korea (NRF) grant funded by the government of Korea (MSIP) (No. 2019R1A2C1010634), the Organelle Network Research Center (NRF2017R1A5A1015366), and KBRI basic research program though the Korea Brain Research Institute funded by the Ministry of Science and ICT (20-BR-02-09).

Acknowledgments: Instrument (transmission electron microscopy and confocal microscopy) data were acquired at the Brain Research Core Facilities in KBRI.

Conflicts of Interest: The authors declare no conflict of interest. 


\section{References}

1. Den Besten, G.; van Eunen, K.; Groen, A.K.; Venema, K.; Reijngoud, D.J.; Bakker, B.M. The role of short-chain fatty acids in the interplay between diet, gut microbiota, and host energy metabolism. J. Lipid Res. 2013, 54, 2325-2340. [CrossRef] [PubMed]

2. Foley, K.A.; MacFabe, D.F.; Vaz, A.; Ossenkopp, K.P.; Kavaliers, M. Sexually dimorphic effects of prenatal exposure to propionic acid and lipopolysaccharide on social behavior in neonatal, adolescent, and adult rats: Implications for autism spectrum disorders. Int. J. Dev. Neurosci. 2014, 39, 68-78. [CrossRef]

3. El-Ansary, A.; Al-Ayadhi, L. Relative abundance of short chain and polyunsaturated fatty acids in propionic acid-induced autistic features in rat pups as potential markers in autism. Lipids Health Dis. 2014, 13, 140. [CrossRef] [PubMed]

4. Nankova, B.B.; Agarwal, R.; MacFabe, D.F.; La Gamma, E.F. Enteric bacterial metabolites propionic and butyric acid modulate gene expression, including CREB-dependent catecholaminergic neurotransmission, in PC12 cells-possible relevance to autism spectrum disorders. PLoS ONE 2014, 9, e103740. [CrossRef]

5. MacFabe, D.F.; Cain, N.E.; Boon, F.; Ossenkopp, K.P.; Cain, D.P. Effects of the enteric bacterial metabolic product propionic acid on object-directed behavior, social behavior, cognition, and neuroinflammation in adolescent rats: Relevance to autism spectrum disorder. Behav. Brain Res. 2011, 217, 47-54. [CrossRef] [PubMed]

6. Duscha, A.; Gisevius, B.; Hirschberg, S.; Yissachar, N.; Stangl, G.I.; Eilers, E.; Bader, V.; Haase, S.; Kaisler, J.; David, C.; et al. Propionic Acid Shapes the Multiple Sclerosis Disease Course by an Immunomodulatory Mechanism. Cell 2020, 180, 1067-1080.e16. [CrossRef]

7. Kim, S.A.; Jang, E.H.; Mun, J.Y.; Choi, H. Propionic acid induces mitochondrial dysfunction and affects gene expression for mitochondria biogenesis and neuronal differentiation in SH-SY5Y cell line. Neurotoxicology 2019, 75, 116-122. [CrossRef]

8. Frye, R.E.; Melnyk, S.; Macfabe, D.F. Unique acyl-carnitine profiles are potential biomarkers for acquired mitochondrial disease in autism spectrum disorder. Transl. Psychiatry 2013, 3, e220. [CrossRef]

9. Tang, Y.; Chen, Y.; Jiang, H.; Nie, D. Short-chain fatty acids induced autophagy serves as an adaptive strategy for retarding mitochondria-mediated apoptotic cell death. Cell Death Differ. 2011, 18, 602-618. [CrossRef]

10. Choi, H.; Kim, I.S.; Mun, J.Y. Propionic acid induces dendritic spine loss by MAPK/ERK signaling and dysregulation of autophagic flux. Mol. Brain 2020, 13, 86. [CrossRef]

11. Fivenson, E.M.; Lautrup, S.; Sun, N.; Scheibye-Knudsen, M.; Stevnsner, T.; Nilsen, H.; Bohr, V.A.; Fang, E.F. Mitophagy in neurodegeneration and aging. Neurochem. Int. 2017, 109, 202-209. [CrossRef] [PubMed]

12. Wang, Y.; Liu, N.; Lu, B. Mechanisms and roles of mitophagy in neurodegenerative diseases. CNS Neurosci. Ther. 2019, 25, 859-875. [CrossRef] [PubMed]

13. Nixon, R.A. The role of autophagy in neurodegenerative disease. Nat. Med. 2013, 19, 983-997. [CrossRef] [PubMed]

14. Chakraborty, J.; Caicci, F.; Roy, M.; Ziviani, E. Investigating mitochondrial autophagy by routine transmission electron microscopy: Seeing is believing? Pharmacol. Res. 2020, 160, 105097. [CrossRef]

15. Jung, M.; Choi, H.; Mun, J.Y. The autophagy research in electron microscopy. Appl. Microsc. 2019, 49, 11. [CrossRef]

16. Katayama, H.; Kogure, T.; Mizushima, N.; Yoshimori, T.; Miyawaki, A. A sensitive and quantitative technique for detecting autophagic events based on lysosomal delivery. Chem. Biol. 2011, 18, 1042-1052. [CrossRef]

17. Sun, N.; Malide, D.; Liu, J.; Rovira, I.I.; Combs, C.A.; Finkel, T. A fluorescence-based imaging method to measure in vitro and in vivo mitophagy using mt-Keima. Nat. Protoc. 2017, 12, 1576-1587. [CrossRef]

18. Allen, G.F.; Toth, R.; James, J.; Ganley, I.G. Loss of iron triggers PINK1/Parkin-independent mitophagy. EMBO Rep. 2013, 14, 1127-1135. [CrossRef]

19. Swanlund, J.M.; Kregel, K.C.; Oberley, T.D. Investigating autophagy: Quantitative morphometric analysis using electron microscopy. Autophagy 2010, 6, 270-277. [CrossRef]

20. Mizushima, N.; Yoshimori, T.; Levine, B. Methods in mammalian autophagy research. Cell 2010, 140, $313-326$. [CrossRef]

21. Gudmundsson, S.; Kahlhofer, J.; Baylac, N.; Kallio, K.; Eskelinen, E.L. Correlative Light and Electron Microscopy of Autophagosomes. Methods Mol. Biol. 2019, 1880, 199-209. [PubMed] 
22. Chen, L.; Ma, K.; Han, J.; Chen, Q.; Zhu, Y. Monitoring Mitophagy in Mammalian Cells. Methods Enzymol. 2017, 588, 187-208.

23. Reddick, L.E.; Alto, N.M. Correlative light and electron microscopy (CLEM) as a tool to visualize microinjected molecules and their eukaryotic sub-cellular targets. J. Vis. Exp. 2012, 63, e3650. [CrossRef] [PubMed]

24. Evans, C.S.; Holzbaur, E.L.F. Quality Control in Neurons: Mitophagy and Other Selective Autophagy Mechanisms. J. Mol. Biol. 2020, 432, 240-260. [CrossRef] [PubMed]

25. Martinez-Vicente, M. Neuronal Mitophagy in Neurodegenerative Diseases. Front. Mol. Neurosci. 2017, 10, 64. [CrossRef]

26. Cheng, A.; Wan, R.; Yang, J.L.; Kamimura, N.; Son, T.G.; Ouyang, X.; Luo, Y.; Okun, E.; Mattson, M.P. Involvement of PGC-1alpha in the formation and maintenance of neuronal dendritic spines. Nat. Commun. 2012, 3, 1250. [CrossRef]

27. Maqsood, R.; Stone, T.W. The Gut-Brain Axis, BDNF, NMDA and CNS Disorders. Neurochem. Res. 2016, 41, 2819-2835. [CrossRef]

28. El-Ansary, A.K.; Al-Daihan, S.K.; El-Gezeery, A.R. On the protective effect of omega-3 against propionic acid-induced neurotoxicity in rat pups. Lipids Health Dis. 2011, 10, 142. [CrossRef]

29. Alfawaz, H.A.; Bhat, R.S.; Al-Ayadhi, L.; El-Ansary, A.K. Protective and restorative potency of Vitamin D on persistent biochemical autistic features induced in propionic acid-intoxicated rat pups. BMC Complement. Altern Med. 2014, 14, 416. [CrossRef]

30. Mauro-Lizcano, M.; Esteban-Martinez, L.; Seco, E.; Serrano-Puebla, A.; Garcia-Ledo, L.; Figueiredo-Pereira, C.; Vieira, H.L.; Boya, P. New method to assess mitophagy flux by flow cytometry. Autophagy 2015, 11, 833-843. [CrossRef]

(C) 2020 by the authors. Licensee MDPI, Basel, Switzerland. This article is an open access article distributed under the terms and conditions of the Creative Commons Attribution (CC BY) license (http://creativecommons.org/licenses/by/4.0/). 